\title{
An Antenna with an Embedded EBG Structure for Non Invasive Hyperthermia Cancer Treatment
}

\author{
Kasumawati Bt Lias, Mohd Zulkarnaen Ahmad Narihan, Norlida Buniyamin
}

\begin{abstract}
This paper describes the development on a microstrip antenna that is built with an electromagnetic band gap (EBG) structure integrated within the antenna. The antenna, termed EBG-microstrip (EBG-M) antenna is purposely built for use in a non-invasive hyperthermia cancer treatment. The EBG-M was built with the aim to improve penetration depth and reduce unwanted hot-spots which may damage surrounding healthy tissues during treatment procedures. In addition, this paper provides an overview of a few salient characteristic of an EBG structure which enables the reduction of the hot-spots. Finally, the results of a simulation using Finite Difference Time Domain (FDTD) that show a few of Specific Absorption Rate (SAR) distribution of the proposed EBG$\mathrm{M}$ antenna are provided. These preliminary results indicate that an EBG-M antenna have an ability to offer penetration depth with minimize unwanted hot spots which are not required for noninvasive hyperthermia cancer treatment.
\end{abstract}

\section{INTRODUCTION}

Hyperthermia is a treatment which requires high temperature around $42^{\circ} \mathrm{C}-45^{\circ} \mathrm{C}$ in order to provide a denaturation onto a cancerous tissue which then will be induced to cell death. Hyperthermia may work alone or commonly as an adjuvant towards chemotherapy and radiotherapy[1]. Hyperthermia can be provided either invasive or non-invasive. An invasive hyperthermia can be utilized by implanting a device in the body or inserting device into a body orifice. Meanwhile, non-invasive hyperthermia cancer treatment is either implemented through single or multiple applicators which will be arranged in array structure. The applicator in this research is referred to an antenna.

In non-invasive hyperthermia cancer treatment, it is offers a cancer procedure with less pain and injury. A noninvasive hyperthermia cancer treatment is applied from outside of the body. If compared to invasive hyperthermia cancer treatment, it is a safer procedure for cancer treatment. However, it is less effective than invasive whereby it is inferior in term of depth penetration effect onto a targeted cancerous tissue. Moreover, non-invasive hyperthermia cancer treatment may also provide severe unwanted hot-spots which may offer damage onto other surrounding healthy tissue. Both of these deficiencies are a significant factors which is requires to be faced for non-invasive hyperthermia cancer treatment. By that, both of these limitations of non-

Kasumawati Bt Lias is with Faculty of Electrical Engineering, UiTM Shah Alam, 40450 Selangor Darul Ehsan, (corresponding author to provide phone: +60198793095; fax: -; e-mail: danza252@ gmail.com).

Mohd Zulkarnaen Bin Ahmad Narihan is with Faculty of Medical and Health Science, Universiti Malaysia Sarawak, 94300 Kota Samarahan, Sarawak (e-mail: anzulkarnaen@fmhs.unimas.my).

Norlida Buniyamin is with Faculty of Electrical Engineering, UiTM Shah Alam, 40450 Selangor Darul Ehsan, (corresponding author to provide phone: +60355442000; fax: -; e-mail: nbuniyamin@salam.uitm.edu.my). invasive hyperthermia cancer treatment need to be improved as to provide safer and effective treatment and should be pondered as one of an alternative treatment apart of chemotherapy, radiotherapy and surgery.

In conjunction with that, this paper main objective is to propose an enhance structure of a microstrip antenna with an EBG structure which will able to improve non-invasive penetration depth and then reduce the unwanted hot-spots in radiating a targeted cancerous tissue for non-invasive hyperthermia cancer treatment.

\section{EBG-M ANTENNA: AN ENHANCED MICROSTRIP ANTENNA FOR NON-INVASIVE HYPERTERMIA CANCER TREATMENT}

Non-invasive hyperthermia cancer treatment is now being a significant cancer treatment to be investigated and studied as this non-invasive hyperthermia cancer treatment may provide safer and effective treatment to fight against various types of cancer. Various types of antennas have been developed and investigated in order to provide non-invasive hyperthermia cancer treatment. Figure 1 shows a progression in antenna development for hyperthermia cancer treatment.

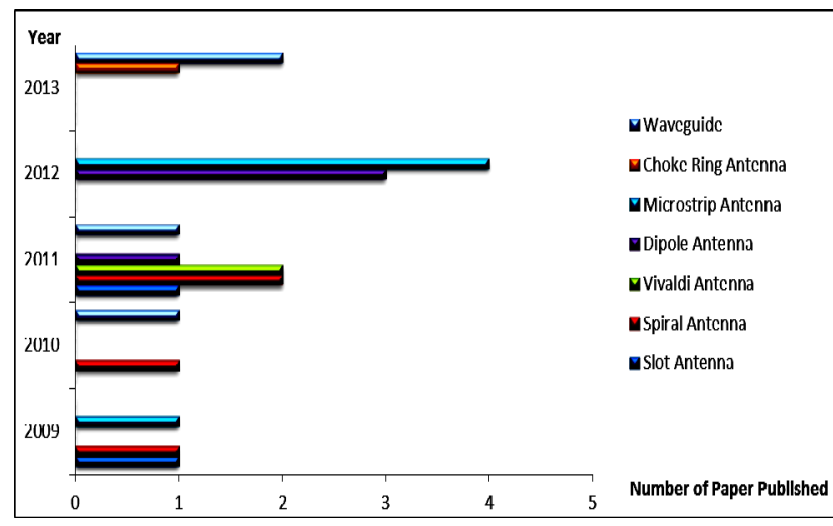

Figure 1: Evolution of Antenna Used for Non-Invasive Hyperthermia Cancer Treatment.

As presented in Figure 1, common types of antennas have been developed and constructed for investigation are slot, dipole, waveguide, spiral, microstrip, vivaldi and choke ring antenna. This is based on information from various sources such as Institute Electrical, Electronics Engineering (IEEE), Medical \& Biological, Engineering \& Computing Journal, International Union of Radio Sciences articles, European Conference on Antennas and Propagation and International Symposium on Antennas and Propagation from year of 2009 until 2013.

From observation in Figure 1, a microstrip antenna has shown the highest numbers of usage with 5 published papers in year of 2009 and 2012 with 1 and 4 published papers, 